\title{
Correction to: Isotopic evolution of prehistoric magma sources of Mt. Etna, Sicily: Insights from the Valle Del Bove
}

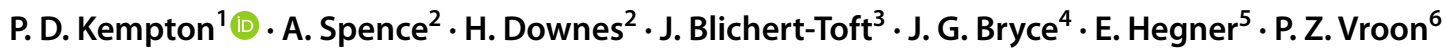

Published online: 27 August 2021

(c) Springer-Verlag GmbH Germany, part of Springer Nature 2021

\section{Correction to: \\ Contributions to Mineralogy and Petrology (2021) 176:56 \\ https://doi.org/10.1007/s00410-021-01804-6}

In the original publication, the names of supplementary files are incorrectly published. The names have been corrected in this correction as below:

A5 frac NNO 4000b 3 $\mathrm{H}_{2} \mathrm{O}$ (XLSX $1490 \mathrm{~kb}$ )

A5 frac QFM 1000b dry (XLSX $2285 \mathrm{~kb}$ )

A5 frac QFM 3500b 4 $\mathrm{H}_{2} \mathrm{O}$ (XLSX $2816 \mathrm{~kb}$ )

A15 frac NNO 4000b $3 \mathrm{H}_{2} \mathrm{O}$ (XLSX $3222 \mathrm{~kb}$ )

A15 frac QFM 1000b dry (XLSX $1941 \mathrm{~kb})$

A15 frac QFM 3500b $4 \mathrm{H}_{2} \mathrm{O}$ (XLSX $2702 \mathrm{~kb}$ )

Online Resource 1 (DOCX $615 \mathrm{~kb}$ )

Online Resource 2 (DOCX $21 \mathrm{~kb}$ )
We apologize for any confusion this may have caused. Results and conclusions in the original paper are not affected by this erratum.

The original article has been corrected.

Supplementary Information The online version contains supplementary material available at https://doi.org/10.1007/s00410-021-01819-z.

Publisher's Note Springer Nature remains neutral with regard to jurisdictional claims in published maps and institutional affiliations.

The original article can be found online at https://doi.org/10.1007/ s00410-021-01804-6.

P. D. Kempton

pkempton@ksu.edu

1 Department of Geology, Kansas State University, Manhattan, KS, USA

2 Department of Earth and Planetary Sciences, Birkbeck University of London, Malet Street, London WC1E 7HX, UK

3 Laboratoire de Géologie de Lyon, CNRS UMR 5276, Ecole Normale Supérieure de Lyon, Université de Lyon, 46 Allée d'Italie, 69007 Lyon, France

4 Department of Earth Sciences, University of New Hampshire, Durham, NH, USA

5 Department of Earth and Environmental Sciences and GeoBioCenter, Ludwig-Maximilians Universität, Munich, Germany

6 Department of Earth Sciences, Faculty of Science, Vrije Universiteit, Amsterdam, The Netherlands 